\title{
Potenciais benefícios do sistema de rastreabilidade animal dos EUA para o setor de carnes americano
}

\author{
Moisés de Andrade Resende Filho ${ }^{1}$
}

Resumo: Este artigo investigou os potenciais ganhos do setor de carnes americano advindos da implantação do Sistema Nacional de Identificação Animal (NAIS), dos EUA. Foram analisados os potenciais efeitos do NAIS sobre a percepção de risco dos consumidores americanos em relação aos perigos decorrentes do consumo das carnes bovina, suína e de aves e seus derivados. Sistemas de equações de demanda foram estimados, incorporando-se como "proxies" da percepção de risco do consumidor, séries de índices de segurança do alimento, separadamente construídas para cada tipo de carne. Tais séries foram concebidas, somando-se o número de referências nos principais jornais americanos a problemas de segurança da carne. Foi utilizado o melhor modelo estimado, escolhido com base em uma série de testes de especificação, para se construir três cenários, simulando-se os casos em que o NAIS não está implementado; que está implementado apenas para o gado bovino; e que está implementado para suínos e bovinos. Foram utilizadas as diferenças entre as receitas estimadas para cada cenário e para cada tipo de carne, como uma medida do potencial ganho advindo da implementação do NAIS. Foi concluído que os setores da carne bovina e suína poderiam arcar com os custos do NAIS. Esse resultado, contudo, depende de o quanto desses potenciais ganhos chegarão efetivamente aos produtores agrícolas.

\footnotetext{
${ }^{1}$ Ph.D. em Economia Aplicada pela University of Minnesota e Professor do Departamento de Análise Econômica da Universidade Federal de Juiz de Fora. E-mail: moises. resende@ufjf.edu.br
} 
Palavras-chave: Sistema nacional de identificação animal; segurança do alimento; sistema de equações de demanda; setor de carnes; EUA.

Abstract: This article investigates the potential gains to the U.S. meat sector with the implantation of the U.S. National Animal Identification System (NAIS). The focus is on the effect that the NAIS could have on consumers' risk perception about eating meat. System of demand equations are estimated using time series of food safety indexes variables used as proxies for consumers' reactions to news on meat safety issues. The series of food safety indexes are built on the basis of the number of food safety news reported in top U.S. newspapers. Using the preferred model estimates, three scenarios are constructed in order to account for the cases wherein the NAIS is not implemented, is implemented only for beef and is implemented for beef and pork. The differences in the estimated total revenues for each scenario are used as a gross measure of the NAIS benefit for each type of meat. The main conclusion is that the gains for the beef and pork sectors would be capable of covering the costs of the NAIS implementation. However, this result depends on how much of these potential gains will arrive at primary producers.

Keywords: Animal identification system; food safety; system of demand equations; meat industry; USA.

Classificação JEL: C22, Q11, Q13, Q18.

\section{Introdução}

Medidas visando à segurança da carne utilizada na alimentação humana têm se fundamentado na premissa de que o perigo de Enfermidades Transmitidas por Alimentos (ETAs) decorre primordialmente da contaminação bacteriana. Com base em tal fato, as inspeções das carnes bovina e de aves nos Estados Unidos da América (EUA) têm, tradicionalmente, focado na detecção e prevenção da contaminação bacteriana em plantas processadoras e empacotadoras e nos locais onde ocorre a preparação de alimentos, como por exemplo, nos restaurantes (Bailey e Slade, 2004). 
Em março de 1996, foi comprovado definitivamente em laboratório a ligação entre a Encefalopatia Espongiforme Bovina (Bovine Spongiform Encephalopathy - BSE daqui em diante), ou mau da vaca louca, e uma nova variante da doença Creutzfeldt-Jakob (vCJD) em humanos (Nardone, 2003). Desde então, a defesa da utilização da rastreabilidade animal como forma de se monitorar a produção na fazenda vem ganhando, cada vez mais, adeptos. Para se entender esse fenômeno, primeiramente, é necessário saber que, diferentemente da contaminação bacteriana, o mau da vaca louca origina-se, exclusivamente, na propriedade agrícola. O fornecimento de rações contendo carne e ossos provenientes de animais contaminados é o principal vetor de contaminação de animais sadios com BSE (Nardone, 2003).

Em caso de ocorrência de um surto de BSE, potenciais tentativas para evitar a entrada de produtos derivados de animais contaminado com BSE na cadeia alimentar humana serão, em geral, limitadas. Isso porque os animais não são usualmente testados para BSE antes que os sintomas da doença se apresentem, tais como: inabilidade do animal em ficar em pé e/ou andar. Até porque, para que se confirme indubitavelmente que um animal está com o mau da vaca louca, é necessário que tecidos de sua massa encefálica sejam testados, o que implica na necessidade de abate do mesmo (Nardone, 2003). Nesse tocante, em caso de detecção de animais infectados com BSE, um sistema nacional de rastreabilidade animal (NAIS) poderia rastrear "para trás" as propriedades por onde tais animais passaram em sua vida para, em seguida, rastrear "para frente" os animais provenientes de tais propriedades. Esse esquema reduziria sobremaneira a quantidade de animais a ser testada para BSE, o que viabilizaria um programa amplo e direcionado de testes. Em última instância, haveria a redução da chance de que subprodutos de animais contaminados com BSE entrassem na cadeia alimentar humana. Adicionalmente, se frigoríficos e rede varejista implementassem sistemas de rastreabilidade capazes de preservar as informações geradas com um sistema nacional de rastreabilidade, poder-se-ia reduzir, ainda mais, as chances de que produtos derivados de animais infectados com BSE chegassem até o consumidor final.

A União Européia e o Japão tornaram a rastreabilidade do gado bovino um bem público, ao obrigarem o uso de sistemas de rastreabilidade 
animal (Souza-Monteiro e Caswell, 2004). Em 2003, toda a importação de carne dos EUA pelo Japão foi embargada após o anúncio do primeiro caso de BSE em uma vaca no estado de Washington. O Japão somente suspendeu esse embargo em dezembro de 2005, condicionado a que os EUA aprimorassem o programa de testes para detecção de BSE e agilizassem a implantação da rastreabilidade da produção de carne bovina. Assim, Brown et al. (2001) defendem que um potencial benefício da implementação de um sistema nacional de rastreabilidade animal seria o maior e mais estável acesso ao mercado internacional.

O fato é que a percepção de risco do consumidor com relação ao mau da vaca louca tem feito com que a rastreabilidade da produção dos animais nas fazendas se tornasse fundamental à sustentação da demanda por carne bovina. Nesse sentido, o primeiro caso confirmado de BSE nos EUA em dezembro de 2003 foi fundamental para acelerar o cronograma da implantação do sistema de rastreabilidade animal dos EUA, denominado de National Animal Identification System (NAIS).

Obviamente, a implementação do NAIS implicará em adicionais custos para os pecuaristas americanos. Como recuperar tais custos ainda é uma questão a ser respondida. Benefícios com o NAIS podem vir dos prêmios em preço que o consumidor final poderia estar disposto a pagar por produtos derivados de animais rastreados. No entanto, resultados conflitantes têm sido obtidos na literatura, vide, por exemplo, os trabalhos de Hobbs (2003) e Dickinson e Bailey (2002).

No presente estudo, assume-se que o benefício do NAIS se daria de forma indireta ao setor que o implementasse, ao propiciar a proteção do mesmo contra eventos e notícias relacionadas a crises de segurança do alimento. Sendo assim, possíveis deslocamentos da curva de demanda para baixo, que ocorreriam em decorrência de um evento ou notícia/ artigo sobre questões relacionadas com a segurança de algum tipo de carne, seriam mitigados se um sistema de rastreabilidade animal, como o NAIS, estivesse em uso. Assim, o presente artigo investiga o quanto poderia ter sido evitado de deslocamentos para baixo nas demandas por carnes bovina e suína nos EUA como um potencial efeito benéfico do NAIS para as cadeias produtivas de cada uma dessas carnes.

O artigo está organizado da seguinte forma: primeiramente são apresentados a estrutura dos sistemas de equações de demanda utiliza- 
dos, os procedimentos adotados para estimá-los e os testes estatísticos empregados no estudo da melhor especificação do sistema. Na seqüência, são explicitados os passos adotados para a coleta dos dados, e, principalmente, a lógica envolvida no cômputo das séries de índices de segurança do alimento para cada tipo de carne. Terceiro, as estimativas obtidas para a melhor especificação do sistema de equações de demanda e as elasticidades com relação à renda, aos preços e os índices de segurança dos alimentos são apresentadas. Finalmente, procede-se à discussão sobre o potencial benefício do NAIS nos EUA, fundamentando-a nos resultados advindos da construção e comparação de cenários.

\section{Metodologia}

Segundo Alston et al. (2001), o uso do modelo Generalized Almost Ideal (GAI) é uma forma de se incorporar, flexível e parcimoniosamente, deslocadores da demanda em sistemas de equações de demanda do tipo Almost Ideal Demand System (AIDS). De acordo com esses mesmos autores, é possível se incorporar deslocadores da demanda em equações de um sistema de equações e, mesmo assim, obter-se estimativas invariantes às unidades de medida utilizadas na mensuração dos preços e quantidades dos produtos analisados.

Piggott e Marsh (2004) utilizaram a estrutura do modelo GAI para incorporar quantidades pré-comprometidas com o consumo de carnes e deslocadores dos interceptos das equações de demanda, de modo a considerarem os efeitos de notícias relacionadas à segurança do alimento sobre a demanda por carnes nos EUA. O presente artigo utiliza os procedimentos e modelos desenvolvidos por Piggott e Marsh (2004), focando adicionalmente sobre notícias relacionadas à BSE. A seguir, apresenta-se a estrutura do modelo originalmente desenvolvido por Piggott e Marsh (2004).

O ponto de partida para a formulação do modelo GAI é a função gasto/dispêndio generalizada, dada por (1).

$$
E(\mathbf{p}, u)=\sum_{i=1}^{N} p_{i} c_{i}+E^{*}(\mathbf{p}, u)
$$

no qual $p_{i}$ é o preço do bem $i$; $c_{i}$ é a quantidade pré-comprometida com 
o consumo do bem $i ; \mathbf{p} \in \Re_{++}^{N}$ é o vetor dos preços dos $N$ bens do grupo carnes; $\sum_{i=1}^{N} p_{i} c_{i}$ representa a parcela do dispêndio pré-comprometida com o consumo dos $N$ bens; e $E^{*}(\mathbf{p}, u)$ é o dispêndio além daquele previamente comprometido com o consumo dos $N$ bens, chamado de dispêndio supernumerário (supernumerary expenditure).

Aplicando-se o lema de Shephard à equação (1) e fazendo-se uso das identidades obtidas com a aplicação da dualidade da teoria do consumidor, obtém-se as funções de demanda Marshallianas, dadas por:

$$
q_{i}=c_{i}+q_{i}^{*}\left(\mathbf{p}, x^{8}\right) \quad \forall i
$$

no qual $q_{i}^{*}\left(\mathbf{p}, x^{*}\right)$ é a função de demanda Marshalliana para o bem $i$; $x^{*}=x-\sum_{i=1}^{n} p_{i} c_{i}$ é o dispêndio supernumerário; e $x$ é o dispêndio total com os $N$ bens.

Pré multiplicando-se (2) por $p_{i} / x$, obtém-se as equações de demanda generalizadas Marshallianas especificadas em termos da parcela do dispêndio total que é gasta com a i-ésima carne $\left(w_{i}\right)$ :

$$
w_{i}=\frac{p_{i} c_{i}}{x}+\frac{x^{*} w_{i}^{*}\left(\mathbf{p}, x^{*}\right)}{x} \quad \forall i
$$

No modelo GAI, $w_{i}^{*}\left(\mathbf{p}, x^{*}\right)$ segue a forma funcional das equações do sistema AIDS (Almost Ideal Demand System), dada por:

$$
w_{i}^{*}\left(\mathbf{p}, x^{*}\right)=\alpha_{i}+\sum_{j=1}^{n} \gamma_{i, j} \ln p_{j}+\beta_{i}\left(\ln x^{*}-\ln a(\mathbf{p})\right) \quad \forall i
$$

no qual $\ln a(\mathbf{p})$ é um índice de preços especificado como uma função translog (5).

$$
\ln a(\mathbf{p})=a_{0}+\sum_{i=1}^{n} \alpha_{i} \ln p_{i}+\frac{1}{2} \sum_{i=1}^{n} \sum_{j=1}^{n} \gamma_{i, j} \ln p_{i} \ln p_{j}
$$

Os deslocadores da demanda são incorporados no modelo GAI, de modo a permitir a introdução de outras variáveis, além dos preços e dispêndios. Seguindo Piggott e Marsh (2004), esses deslocadores da demanda são introduzidos no modelo via modificação das quantidades pré-comprometidas com o consumo de cada carne. Para tanto, redefinese o termo $c_{i}$ na equação (3) para: 


$$
c_{i}=c_{i, 0}+\tau_{i} t+\sum_{k=1}^{3} \theta_{i, k} D_{k}+\sum_{m=0}^{L} \phi_{i, m} b f_{t-m}+\pi_{i, m} p k_{t-m}+\kappa_{i, m} p y_{t-m} \quad \forall i
$$

no qual $t$ é a variável tendência; $D_{k}$ representa variáveis dicotômicas (dummies) sazonais para a demanda trimestral de cada tipo de carne; $b f_{t-m}$ é a variável índice agregado de segurança do alimento para a carne bovina com defasagem temporal $m ; p k_{t-m}$ é a variável índice agregado de segurança do alimento para a carne suína com defasagem temporal $m$; e , finalmente, $p y_{t-m}$ é a variável índice agregado de segurança do alimento para a carne de aves com defasagem temporal $m$. Esses índices serão propriamente definidos na seção 3 deste artigo.

A duração de tempo em que um evento ou notícia/artigo relacionado a problemas com a segurança dos alimentos permanece afetando a demanda por carne bovina, suína e de aves é tratado no modelo como uma incógnita. Ou seja, são realizados testes de especificação de modo a se determinar o valor de $L$. Para tanto, serão testados valores de $L=$ $\{0,1$ e 2$\}$.

Restrições advindas da teoria do consumidor foram impostas aos parâmetros dos modelos, seguindo o que fizeram e sugerem Fisher et al. (2001) e Piggott e Marsh (2004). Tais restrições são derivadas da premissa de que o sistema de equações de demanda deve satisfazer as condições: adding-up (toda a renda disponível é gasta), homogeneidade de grau zero nos preços e renda (ausência de ilusão monetária) e simetria da matriz de substituição de Slutsky (aplicação do teorema de Young). Assim sendo, as restrições de homogeneidade, simetria e adding-up são impostas ao sistema de equações respectivamente por (7), (8) e (9).

$$
\begin{aligned}
& \sum_{j=1}^{n} \gamma_{i, j}=0 \quad \forall i \\
& \gamma_{i, j}=\gamma_{j, i} \quad \forall i, j \\
& \sum_{i=1}^{n} \alpha_{i}=1, \sum_{i=1}^{n} \beta_{i}=0, \mathrm{e} \sum_{i=1}^{n} \gamma_{i, j}=0 \forall j
\end{aligned}
$$

Sabe-se que as parcelas do dispêndio total com carnes devem somar um, ou seja, toda a renda disponível para o consumo de carnes é 
necessariamente gasta (condição adding-up). Como conseqüência disso, a matriz de covariância do sistema de equações, quando estimado incluindo-se todas as equações, seria necessariamente singular. Para contornar tal problema, a equação referente à parcela do orçamento gasto com aves é retirada do sistema. Assim, as estimativas para os parâmetros da equação para carne de aves podem ser obtidas, após a estimação do sistema, via aplicação das restrições (7), (8) e (9).

Em estudos com séries temporais, a ocorrência de autocorrelação ou correlação serial dos erros é um problema recorrente. Sabe-se que em presença de autocorrelação as estimativas dos parâmetros permanecem consistentes e não-viesados, mas as estimativas das variâncias dos parâmetros passam a ser viesadas e inconsistentes (Berndt, 1996).

Fisher et al. (2006) assumem a presença de autocorrelação e, assim, já impõem a priori correções para correlação serial de primeira ordem dos erros nos sistemas de equações não-lineares de demanda estimados por eles. No presente artigo, ao contrário do que fizeram Fisher et al. (2006), foi investigado se há a ocorrência de autocorrelação e, para tanto, testa-se duas formas alternativas de correção do problema. Desta forma, os modelos econométricos são estimados segundo o sistema de equações (10), que segue a transformação para correção da autocorrelação, pioneiramente proposta por Berndt e Savin (1975).

$$
W_{t}=\mathrm{R} W_{t-1}+\Upsilon_{t} C_{t}-\mathrm{Rr}_{t-1} C_{t-1}+\frac{x_{t}^{*}}{x_{t}} W_{t}^{*}\left(\mathbf{p}_{t}, x_{t}^{*}\right)-\mathrm{R} \frac{x_{t-1}^{*}}{x_{t-1}} W_{t-1}^{*}\left(\mathbf{p}_{t-1}, x_{i-1}^{*}\right)
$$

no qual

$$
W_{t} \equiv\left(\begin{array}{c}
w_{b, t} \\
w_{p, t}
\end{array}\right), \mathrm{R} \equiv\left(\begin{array}{cc}
\rho_{b, b} & \rho_{b, p} \\
\rho_{p, b} & \rho_{p, p}
\end{array}\right), \Upsilon_{t} \equiv\left(\begin{array}{cc}
\frac{p_{b, t}}{x_{t}} & 0 \\
0 & \frac{p_{p, t}}{x_{t}}
\end{array}\right) \text { e } C_{t} \equiv\left(\begin{array}{c}
c_{b, t} \\
c_{p, t}
\end{array}\right)
$$

com os subscritos $b$, p e c denotando carne bovina, suína e de aves;

$W_{t}^{*}\left(\mathbf{p}_{t}, x_{t}^{*}\right)=\left(\begin{array}{c}w_{b, t}^{*}\left(\mathbf{p}_{t}, x_{t}^{*}\right) \\ w_{p, t}^{*}\left(\mathbf{p}_{t}, x_{t}^{*}\right)\end{array}\right)$ com $w_{i, t}$ representando a parcela observada do gasto com carnes que é utilizada no consumo da carne $i ; p_{i, t}$, representa os preços observados para a carne $i$ no tempo $t$; $c_{i, t}$ representa as quantidades pré-comprometidas com a carne $i$, como definido pela equação (6); e $w_{i, t}^{*}\left(\mathbf{p}_{t}, x_{t}^{*}\right)$ é especificada segundo a equação (4), utilizando-se 
para tanto a série do dispêndio supernumerário $x_{t}{ }_{t}=x_{t}-\sum_{i=1}^{n} p_{i t} c_{i t}$.

No sentido de testar e corrigir a autocorrelação, a matriz $\mathrm{R}$ do sistema de equações (10) a ser estimado é especificada de três maneiras alternativas: a) como uma matriz nula ( $\mathrm{N}-\mathrm{R}^{\text {matriz }}$ ), em que todos os elementos são iguais a zero, o que implica dizer que nenhuma correção para autocorrelação está sendo feita; b) como uma matriz Diagonal (D$\mathrm{R}^{\text {matriz}}$ ), no qual as estimativas dos elementos da diagonal principal são forçados a serem iguais e os demais elementos da matriz são, forçosamente, nulos; c) como uma matriz (F-R ${ }^{\text {matriz }}$ ), em que as estimativas dos seus componentes podem assumir qualquer valor real, sem qualquer restrição.

\subsection{Procedimentos para estimação e testes das especificações das equações do sistema}

Todos os sistemas de equações foram estimados utilizando-se dois algoritmos do tipo Full Information Maximum Likelihood (FIML), disponibilizados no programa EViews, quais sejam: o algoritmo Berndt, Hall, Hall, e Hausman (BHHH) para problemas de máximo verossimilhança e algoritmo de Marquardt (detalhes técnicos podem ser obtidos em Quantitative Micro Software, 2004). Foram utilizados diferentes valores iniciais para os parâmetros a serem estimados e uma combinação desse dois algoritmos, de forma a aumentar a chance de se obter o máximo global para a função multivariada de verossimilhança. Adicionalmente, sabe-se que os estimadores do tipo FIML são assintoticamente eficientes para modelos simultâneos lineares e não-lineares, admitindo-se que os erros contemporâneos são conjuntamente normalmente distribuídos (Quantitative Micro Software, 2004).

Sabe-se que testes estatísticos assintóticos, como o teste da razão de verossimilhança (daqui em diante teste $L R$ ), tendem a rejeitar excessivamente as restrições impostas a um sistema de equações obtido com o uso de amostras finitas de dados (Moschini et al., 1994). Segundo os mesmos autores, não existe um consenso sobre a melhor forma de se corrigir a estatística do teste $L R$. Contudo, ao estudarem o método proposto originalmente por Italianer (1985), encontraram bons resultados 
quando aplicado a sistemas de equações não-lineares, como os sistemas de equações estimados neste artigo. Dessa forma, seguindo a sugestão de Moschini et al. (1994), os testes de razão de verossimilhança foram executados ajustando-se a estatística do teste $L R$ convencional segundo a equação (11).

$$
L R s=\left(\left(M T-0,5\left(\left(k^{u}+k^{r}\right)-M(M+1)\right)\right) / M T\right) L R
$$

no qual $M$ é o número de equações estimadas no sistema, $T$ é o tamanho da amostra, $k^{u}$ é o número de parâmetros no modelo irrestrito; $L R$ $=2\left(L L^{U}-L L^{R}\right)$, onde $L L^{U}$ e $L L^{R}$ são os logaritmos dos valores máximos da função máximo verossimilhança obtidos respectivamente para os modelos irrestritos e restritos.

A estatística ajustada do teste de razão de verossimilhança segue assintoticamente uma distribuição Qui-Quadrado com graus de liberdade igual ao número de parâmetros adicionalmente incorporados no modelo irrestrito em comparação ao modelo restrito. A hipótese nula é de que todos os parâmetros adicionais são conjuntamente nulos.

\section{Dados e construção das séries}

Doze especificações do sistema de equações de demanda (10) foram estimadas,_utilizando dados trimestrais de 1982(1) a 2001(1), o que resultou num total de 77 observações. O tamanho das séries se mostrou suficiente para a obtenção de estimativas razoáveis para os sistemas de equações, tanto do ponto de vista estatístico, como do ponto de vista da teoria econômica (teoria do consumidor). Além disso, a utilização de séries de tempo mais longas poderia colocar em cheque a premissa de que as elasticidades permanecem constantes em todo o período de tempo analisado.

As séries do consumo aparente per capita ${ }^{2}$ para cada carne foram coletadas no the United States Department of Agriculture/Economic Re-

\footnotetext{
${ }^{2} \mathrm{O}$ consumo aparente per capita da carne $i$ é calculado somando-se, para a carne em questão, a produção, os estoques iniciais do período e as importações e subtraindo-se os estoques finais e as exportações. Em seguida, o valor obtido é convertido para o peso relativo ao varejo e dividido pela população residente do período.
} 
search Service (USDA/ERS, 2005a e 2005b) e estão mensuradas na base do peso no varejo em libras de carne bovina, suína, de frango e peru. As séries de preços foram também coletadas em USDA/ERS (2005a e 2005b) e os preços são em dólares americanos por libra-peso, relativos ao varejo. A série de preços da carne bovina corresponde à série Choice. Para o preço da carne suína, a série utilizada foi a Retail Pork Value. Já para o frango, a série de preços utilizada foi a Whole Fryers Retail e, finalmente, para os preços da carne de peru, a série usada foi a Whole Frozen Birds.

Como sugerido por Piggott e Marsh (2004), a série de consumo aparente per capita de carne de aves foi construída, somando-se as séries de consumo aparente per capita de frango e peru. Já a série de preços para a carne de aves foi construída como uma média ponderada dos preços do frango e peru. Para tanto, somou-se, para cada período de tempo, os preços das carnes de frango e de peru ponderados por suas respectivas quantidades para, em seguida, dividir o valor resultante pela soma do consumo aparente per capita de peru e frango.

Séries de índices de segurança do alimento foram construídas contabilizando-se o número de reportagens e notícias veiculadas na imprensa escrita, relacionadas a cada questão envolvendo problemas de segurança no consumo de cada tipo de carne. Para tanto, foram contabilizados o número de vezes em que cada palavra-chave relacionada a um problema de falta de segurança era mencionada em artigos publicados nos cinqüenta principais jornais de língua inglesa em circulação no Meio Oeste, Nordeste, Leste e Sudeste dos EUA para cada carne e para todo o período considerado, 1982(1) a 2001(1). Na coleta dessas informações, foi utilizada a versão acadêmica da ferramenta de busca Lexis-Nexis (disponível em http://web.lexis-nexis.com/universe/).

É importante ressaltar que a unidade de medida é a referência a uma palavra-chave que serve para retratar um problema de segurança do alimento relacionado a um tipo de carne. Dessa forma, é possível encontrar duas ou mais referências para distintos problemas de segurança do alimento em um mesmo artigo de jornal. Por exemplo, é possível que um mesmo artigo mencione as palavras-chaves BSE e E. Coli, sendo assim contabilizados duas vezes.

As buscas foram realizadas de tal maneira que artigos que tratavam 
de problemas de segurança do alimento eram separadamente contabilizados para cada tipo de carne, obtendo-se, assim, três séries trimestrais de índices de segurança do alimento, uma para cada tipo de carne. Dois grupos de índices de segurança do alimento foram gerados. O primeiro grupo de índices contabiliza toda referência na mídia escrita a problema de segurança da carne, considerado como "aparentemente não-relacionados com o NAIS". O segundo grupo compreende todas as referências na mídia escrita a problemas de segurança do alimento que poderiam, em tese, ser atenuados, caso o NAIS já estivesse em uso. Dessa forma, as séries do segundo grupo são denominadas de séries de índices de segurança do alimento relacionada com o NAIS. Para esse segundo grupo, considerou-se que o NAIS teria efeito atenuante sobre a percepção de risco dos consumidores de carne se a referência tratasse de $\mathrm{BSE}^{3}$.

Assim, duas rodadas de pesquisa foram efetuadas. Na primeira rodada, foi realizado um conjunto de consultas, utilizando-se a ferramenta Lexis-Nexis, com vistas a se coletar os dados para as séries de segurança do alimento aparentemente não-relacionadas com o NAIS. Assim, foram realizadas buscas, utilizando-se as seguintes palavras chaves: food safety, contamination, product recall, outbreak, salmonella, listeria, E. coli, trichinae, staphylococcus ou foodborne. Cada busca é subseqüentemente refinada, de modo a permitir agrupar os resultados obtidos por tipo de carne. Dessa forma, são realizadas três consultas dentro do conjunto de resultados obtidos no primeiro passo, utilizandose, para tanto, três conjuntos de palavras chaves: (a) beef or hamburger, (b) pork or ham, e (c) chicken or turkey or poultry. As três séries de índices obtidos, uma para cada tipo de carne, serão tratadas, daqui em diante, como índices de segurança do alimento aparentemente nãorelacionados com o NAIS.

A segunda rodada de buscas objetivou encontrar referências a problemas de segurança do alimento que poderiam ser atenuados, caso o NAIS estivesse em uso. Dessa forma, buscas foram conduzidas, utilizado-se como palavras chaves: BSE or Bovine Spongiform Encephalopathy

\footnotetext{
${ }^{3}$ Optou-se por contabilizar apenas as referências à BSE devido ao fato de se ter observado um número muito pequenos de referências a outras questões que poderiam estar ligadas ao NAIS, tais como: zoonoses e resíduos químicos de medicamentos e drogas em carnes.
} 
or Mad Cow. Seguindo a mesma lógica utilizada na construção dos índices de segurança do alimento aparentemente não-relacionados com o NAIS, os resultados foram refinados, de modo a permitir a coleta do número de referências separadamente para cada tipo de carne. Dessa forma, as três séries de índices resultantes dessa segunda rodada de buscas são chamadas de índices de segurança do alimento relacionadas com o NAIS.

Finalmente, três séries de índices, chamadas de índices agregados de segurança do alimento, foram construídas somando-se as séries de índices aparentemente não-relacionados com o NAIS com a série de índices de segurança do alimento relacionada com o NAIS, para cada tipo de carne.

\section{Resultados e discussão}

Foram estimadas doze diferentes especificações para o sistema de equações (10), considerando-se: três estruturas de correções para a autocorrelação dos erros (N-R ${ }^{\text {matriz }}, \mathrm{D}-\mathrm{R}^{\text {matriz }}$ e $\left.\mathrm{F}-\mathrm{R}^{\text {matriz }}\right)$, a não inclusão das séries de índices agregados de segurança do alimento (No-FS), a inclusão das séries de índices agregados de segurança do alimento sem defasagem $(L=0)$, com defasagem de 1 período de tempo $(L=1)$ e com defasagem de 2 períodos de tempo $(L=2)$.

Os resultados dos testes estatísticos utilizados para a determinação da especificação mais apropriada para o sistema de equações são apresentados na Tabela 1 . 
Tabela 1. Testes de hipóteses para a significância das variáveis índices agregados de segurança do alimento e para as correções de autocorrelação

\begin{tabular}{|c|c|c|c|c|c|c|c|}
\hline \multirow[b]{3}{*}{ Modelo } & \multicolumn{3}{|c|}{$\begin{array}{l}\text { Tamanho da Defasagem } \\
\text { dos Índices de Segurança } \\
\text { do Alimento }\end{array}$} & & \multicolumn{3}{|c|}{ Correções para Autocorrelação } \\
\hline & $\mathrm{H}_{0}:$ No-FS & $\mathrm{H}_{0}: \mathrm{L}=0$ & $\mathrm{H}_{0}: \mathrm{L}=1$ & & $\mathrm{H}_{0}: \mathrm{N}-\mathrm{R}^{\text {matriz }}$ & $\mathrm{H}_{0}: \mathrm{D}-\mathrm{R}^{\text {matriz }}$ & $\mathrm{H}_{0}: \mathrm{N}-\mathrm{R}^{\text {matriz }}$ \\
\hline & $\mathrm{H}_{\mathrm{a}}: \mathrm{L}=0$ & $\mathrm{H}_{\mathrm{a}}: \mathrm{L}=1$ & $\mathrm{H}_{\mathrm{a}}: \mathrm{L}=2$ & Modelo & $\mathrm{H}_{\mathrm{a}}: \mathrm{D}-\mathrm{R}^{\text {matriz }}$ & $\mathrm{H}_{\mathrm{a}}: \mathrm{F}-\mathrm{R}^{\text {matriz }}$ & $\mathrm{H}_{\mathrm{a}}: \mathrm{F}-\mathrm{R}^{\text {matriz }}$ \\
\hline $\mathrm{N}-\mathrm{R}^{\text {matriz }}$ & $28,500^{*}$ & 10,517 & 12,380 & No-FS & $19,422^{*}$ & 4,745 & $24,484^{*}$ \\
\hline $\mathrm{D}-\mathrm{R}^{\text {matriz }}$ & $17,821^{*}$ & 12,221 & 13,760 & $\mathrm{~L}=0$ & $8,467^{*}$ & 1,182 & $9,552^{*}$ \\
\hline \multirow[t]{2}{*}{ F-R ${ }^{\text {matriz }}$} & 14,088 & 12,469 & 13,540 & $\mathrm{~L}=1$ & $9,586^{*}$ & 1,636 & $11,105^{*}$ \\
\hline & & & & $\mathrm{L}=2$ & $10,276^{*}$ & 2,230 & $12,372^{*}$ \\
\hline g.l. & 9 & 9 & 9 & & 1 & 3 & 4 \\
\hline$\chi_{5 \%, g .1 .}^{2}$ & 16,919 & 16,919 & 16,919 & & 3,841 & 7,815 & 9,488 \\
\hline
\end{tabular}

Nota: Um * denota a rejeição de $\mathrm{H}_{0}$ ao nível de $5 \%$; L representa a defasagem máxima das séries de índices de segurança do alimento incluídos nos modelos; No-FS indica que o modelo foi estimado sem a incorporação dos índices agregados de segurança do alimento como regressores; g.l. denota graus de liberdade. Os valores apresentados são as estatísticas calculadas para os testes ajustados de razão de verossimilhança calculados segundo equação (11).

O primeiro passo na busca da melhor especificação do sistema, será avaliar a necessidade de se utilizar correções para a violação do pressuposto de ausência correlação serial dos erros. Tomando-se os resultados apresentados na Tabela 1, colunas de 6 a 8 para as linhas No-FS, $L=0, L=1$ e $L=2$ verifica-se o seguinte: para todos os grupos de modelos, os resultados dos testes de hipóteses indicam a seguinte ordem de preferência ${ }^{4}: \mathrm{D}-\mathrm{R}^{\text {matriz }} \succ \mathrm{N}-\mathrm{R}^{\text {matriz }}, \mathrm{D}-\mathrm{R}^{\text {matriz }} \succ \mathrm{F}-\mathrm{R}^{\text {matriz }}$ e $\mathrm{F}-\mathrm{R}^{\text {matriz }} \succ \mathrm{N}-\mathrm{R}^{\text {matriz }}$. Com isso, a ordem final de preferência dentre qualquer uma das quatro classes de modelos (No-FS, $\mathrm{L}=0, \mathrm{~L}=1$ e $\mathrm{L}=2$ ) é: $\mathrm{D}-\mathrm{R}$ matriz $\succ \mathrm{F}-\mathrm{R}^{\text {matriz }} \succ \mathrm{N}$ $\mathrm{R}^{\text {matriz }}$. Em suma, detectou-se que há ocorrência de correlação serial de primeira ordem, mas que a estimação do sistema (10), utilizando-se uma matriz diagonal ( $D-R^{\text {matriz }}$ ), seria suficiente para corrigir tal problema.

O segundo passo nos testes de especificação é detectar o número ótimo de defasagens $(L)$ a ser utilizado para as variáveis índices agregados de segurança do alimento. Para tanto, utiliza-se o resultado obtido no primeiro passo, considerando-se apenas as especificações do sistema

${ }^{4} \mathrm{O}$ símbolo $\succ$ significa "preferido a". 
em que a matriz diagonal, D-R ${ }^{\text {matriz }}$, é utilizada. Assim, avaliando os resultados dos testes apresentados na Tabela 1, colunas 2 a 4, referentes à linha D-R ${ }^{\text {matriz }}$, observa-se o seguinte: primeiro, a inclusão dos índices de segurança do alimento é necessária, uma vez que $\mathrm{H}_{0}$ : No-FS é rejeitado contra $\mathrm{H}_{\mathrm{a}}$ : $L=0$; segundo, é suficiente incluir os índices agregados de segurança do alimento sem nenhuma defasagem temporal, uma vez que $\mathrm{H}_{0}: L=0$ não foi rejeitado contra $\mathrm{H}_{\mathrm{a}}: L=1$ e, finalmente, $\mathrm{H}_{0}: L=1$ não foi rejeitado contra $\mathrm{H}_{\mathrm{a}}: L=2$. Dessa forma, o resultado final para a ordem de preferência dos modelos estimados, utilizando-se uma matriz diagonal (D-R ${ }^{\text {matriz }}$ ), é: $\mathrm{L}=0 \succ$ No-FS e $\mathrm{L}=0 \succ \mathrm{L}=1 \succ \mathrm{L}=2$. Assim, os testes estatísticos da razão de verossimilhança ajustada levam à conclusão de que, na melhor especificação do sistema, os índices agregados de segurança do alimento devem ser incluídos sem defasagem temporal nos modelos estimados, utilizando-se a matriz D-R matriz. Sendo assim, essa especificação do sistema é a escolhida.

A Tabela 2 apresenta as estimativas do sistema (10) escolhido. Ou seja, o sistema (10) estimado com a utilização da matriz D-R matriz e incluindo os índices agregados de segurança do alimento sem nenhuma defasagem. 
Tabela 2. Estimativas dos parâmetros do sistema de equações (10), estimado com a utilização da matriz D-R ${ }^{\text {matriz }}$ e com a inclusão dos índices agregados de segurança do alimento sem defasagem de tempo

\begin{tabular}{|c|c|c|c|c|c|}
\hline Parâmetro & & Parâmetro & & Parâmetro & \\
\hline$\rho$ & $\begin{array}{c}0,3200 * * \\
(0,1448)\end{array}$ & $\theta_{p, 2}$ & $\begin{array}{c}-1,5050 * * \\
(0,1819)\end{array}$ & $\kappa_{b, 0}$ & $\begin{array}{c}0,0010 \\
(0,0018)\end{array}$ \\
\hline $\mathrm{c}_{\mathrm{b}, 0}$ & $\begin{array}{c}15,4343^{* *} \\
(3,1613)\end{array}$ & $\theta_{p, 3}$ & $\begin{array}{c}-1,1668^{* *} \\
(0,1593)\end{array}$ & $\kappa_{p, 0}$ & $\begin{array}{c}0,0018 \\
(0,0020)\end{array}$ \\
\hline $\mathrm{C}_{\mathrm{p}, 0}$ & $\begin{array}{c}3,7411 \\
(4,5608)\end{array}$ & $\theta_{\mathrm{c}, 1}$ & $\begin{array}{c}-2,4355^{* *} \\
(0,1843)\end{array}$ & $\kappa_{\mathrm{c}, 0}$ & $\begin{array}{l}-0,0005 \\
(0,0027)\end{array}$ \\
\hline $\mathrm{C}_{\mathrm{c}, 0}$ & $\begin{array}{c}0,5639 \\
(13,9236)\end{array}$ & $\theta_{\mathrm{c}, 2}$ & $\begin{array}{c}-1,7224 * * \\
(0,2404)\end{array}$ & $\alpha_{0}$ & $\begin{array}{c}9,0710 \\
(9,5771)\end{array}$ \\
\hline$\tau_{b}$ & $\begin{array}{c}0,0425^{* *} \\
(0.0216)\end{array}$ & $\theta_{c, 3}$ & $\begin{array}{c}-1,2922^{* *} \\
(0,2280)\end{array}$ & $\alpha_{b}$ & $\begin{array}{c}3,3196 \\
(4,7797)\end{array}$ \\
\hline$\tau_{\mathrm{p}}$ & $\begin{array}{c}0,0763 * * \\
(0,0208)\end{array}$ & $\phi_{\mathrm{b}, 0}$ & $\begin{array}{l}-0,0012^{*} \\
(0,0007)\end{array}$ & $\alpha_{p}$ & $\begin{array}{l}-0,7803 \\
(1,7030)\end{array}$ \\
\hline$\tau_{\mathrm{c}}$ & $\begin{array}{c}0,1732 * * \\
(0,0269)\end{array}$ & $\phi_{\mathrm{p}, 0}$ & $\begin{array}{c}-0,0017^{* *} \\
(0,0007)\end{array}$ & $\gamma_{\mathrm{bb}}$ & $\begin{array}{c}2,9872 \\
(2,9241)\end{array}$ \\
\hline$\theta_{\mathrm{b}, 1}$ & $\begin{array}{c}0,0044 \\
(0,1956)\end{array}$ & $\phi_{\mathrm{c}, 0}$ & $\begin{array}{l}-0,0011^{*} \\
(0,0006)\end{array}$ & $\gamma_{\mathrm{bp}}$ & $\begin{array}{c}-0,8510 \\
(1,1712)\end{array}$ \\
\hline$\theta_{\mathrm{b}, 2}$ & $\begin{array}{c}0,5749 * * \\
(0,2350)\end{array}$ & $\pi_{\mathrm{b}, 0}$ & $\begin{array}{c}0,0018 \\
(0,0043)\end{array}$ & $\gamma_{\mathrm{pp}}$ & $\begin{array}{c}0,3219 \\
(0,4059)\end{array}$ \\
\hline$\theta_{\mathrm{b}, 3}$ & $\begin{array}{c}0,8705^{* *} \\
(0.1595)\end{array}$ & $\pi_{p, 0}$ & $\begin{array}{c}0,0008 \\
(0,0060)\end{array}$ & $\beta_{\mathrm{b}}$ & $\begin{array}{c}0,6049 * * \\
(0,2775)\end{array}$ \\
\hline$\theta_{p, 1}$ & $\begin{array}{c}-1,0305^{* *} \\
(0,1390)\end{array}$ & $\pi_{\mathrm{c}, 0}$ & $\begin{array}{c}0,0039 \\
(0,0087)\end{array}$ & $\beta_{p}$ & $\begin{array}{c}-0,2032^{* *} \\
(0,080)\end{array}$ \\
\hline Log Likelihood & 662,6353 & $\mathrm{R}^{2}$ beef & 0,9865 & $\mathrm{R}^{2}$ pork & 0,9305 \\
\hline
\end{tabular}

Nota: números em parênteses são os erros padrão estimados; ${ }^{* *}$ representa coeficientes que foram estatisticamente diferentes de zero ao nível de $5 \%$ pelo teste $z$; * representa coeficientes que foram estatisticamente diferentes de zero ao nível de $10 \%$ pelo teste $z$; beef denota a equação para carne bovina e pork denota a equação para carne de porco; $\rho$ é o elemento da diagonal da matriz D-R ${ }^{\text {matriz }} ; \mathrm{c}_{\mathrm{b}, 0}, \mathrm{c}_{\mathrm{p}, 0}$ e $\mathrm{c}_{\mathrm{c}, 0} \mathrm{~s}$ ão interceptos; $\tau_{\mathrm{b}}$, $\tau_{\mathrm{p}}$, e $\tau_{\mathrm{c}}$ são os coeficientes associados a variável tendência da quantidades pré-comprometidas respectivamente com carne bovina, carne suína e carne de aves; $\theta_{\mathrm{b}, 1}, \theta_{\mathrm{b}, 2}$ e $\theta_{\mathrm{b}, 3}$ são os coeficientes da primeira, segunda e terceira variável binária sazonal na equação para a quantidade pré-comprometida com carne bovina; 
$\theta_{\mathrm{p}, 1}, \theta_{\mathrm{p}, 2}$ e $\theta_{\mathrm{p}, 3}$ são os coeficientes da primeira, segunda e terceira variável binária sazonal na equação para a quantidade pré-comprometida com carne suína; $\theta_{c, 1}, \theta_{c, 2}$ e $\theta_{c, 3}$ são os coeficientes da primeira, segunda e terceira variável binária sazonal na equação para a quantidade pré-comprometida com carne de aves; $\phi_{\mathrm{b}, 0}, \pi_{\mathrm{b}, 0}$ e $\kappa_{\mathrm{b}, 0}$ são respectivamente os coeficientes para os índices de segurança do alimento para carne bovina, suína e de aves com defasagem zero na equação da quantidade précomprometida com carne bovina; $\phi_{\mathrm{p}, 0}, \pi_{\mathrm{p}, 0}$ e $\kappa_{\mathrm{p}, 0}$ são respectivamente os coeficientes para os índices de segurança do alimento para carne bovina, suína e de aves com defasagem zero na equação da quantidade pré-comprometida com carne de frango; $\alpha_{0}$ é o intercepto da equação do índice de preço translog; $\alpha_{b}$ e $\alpha_{p}$ são os interceptos respectivamente para as equações da parcela do gasto com carnes gasta com carne bovina e carne suína: $\gamma_{\mathrm{bb}}, \gamma_{\mathrm{bp}}$ e $\gamma_{\mathrm{pp}}$ são os coeficientes das equações na forma AIDS; $\beta_{\mathrm{b}}$ e $\beta_{\mathrm{p}}$ são os coeficientes associados as variáveis log natural do gasto real com carnes, respectivamente nas equações da parcela do orçamento (budget share) na forma AIDS para a carne bovina e suína.

Dado os resultados apresentados na Tabela 2, verifica-se que as estimativas de todos os interceptos das quantidades pré-comprometidas com carne bovina, suína e de aves $\left(c_{b, 0}, c_{p, 0}, c_{c, 0}\right)$ são não-negativas, como esperado. Contudo, apenas o coeficiente da quantidade pré-comprometida com carne bovina foi estatisticamente significativo pelo teste $\mathrm{z}$ a $5 \%$ de probabilidade. Os coeficientes associados com as variáveis tendências $\left(\tau_{i} \forall i\right)$ mostraram-se todos individualmente significativos pelo teste $\mathrm{z}$ a $5 \%$, indicando a importância de se incluir essas variáveis nas equações do modelo. Exceto pelo coeficiente da primeira variável dummy para a equação da quantidade pré-comprometida com carne bovina $\theta_{b, 1}$, os coeficientes de todas as demais variáveis dummies sazonais $\left(\theta_{i, 1}, \theta_{i, 2}, \theta_{i, 3} \forall i\right)$ foram individualmente e estatisticamente significativos ao nível de $5 \%$ pelo teste $\mathrm{z}$, o que confirma a necessidade de inclusão das dummies sazonais nas equações do sistema.

Como a priori esperado, o impacto das notícias relacionadas à segurança do alimento sobre o consumo pré-comprometido com uma carne deve ser negativo, se a notícia foi veiculada no contexto da carne em questão. Dessa forma, seria esperado que as estimativas dos coeficientes $\phi_{b, 0}, \pi_{p, 0}$ e $\kappa_{c, 0}$ fossem negativas, o que não é confirmado, por conta das estimativas positivas para $\pi_{p, 0}$ e $\kappa_{c, 0}$. Deve-se observar, contudo, que a estimativa do parâmetro $\phi_{b, 0}$ que ${ }_{b}$ foi negativa, foi a única individualmente e estatisticamente diferente de zero pelo teste z a $10 \%$.

Exceto por $\phi_{c, 0}$ e $\phi_{p, 0}$ todos os demais coeficientes, associados aos 
índices agregados cruzados de segurança do alimento, não diferem estatisticamente e individualmente de zero pelo teste $\mathrm{z}$ a $10 \%$. Como as suas estimativas são números reais negativos, conclui-se que as referências na mídia escrita sobre a segurança das carnes de porco e de aves afetam adversamente as quantidades pré-comprometidas com carne bovina (spillover effect). A despeito da grande maioria dos coeficientes para as variáveis índices agregados de segurança do alimento não serem individualmente e estatisticamente significativos, essas variáveis são mantidas nas equações do sistema, uma vez que se apresentaram conjuntamente significativos, como indicaram a bateria de testes de especificação apresentados na Tabela 1.

Finalmente, observa-se na Tabela 2, que todas as equações do sistema escolhido apresentaram excelente ajuste, considerando-se os coeficiente de ajustamento ou $\mathrm{R}^{2}$ das equações da carne bovina e suína. A seguir, as estimativas das elasticidades para o sistema escolhido serão apresentadas e avaliadas.

As elasticidades foram calculadas para o sistema escolhido e correspondem às médias amostrais das elasticidades computadas para cada observação da amostra, utilizando-se as parcelas estimadas do gasto com cada tipo de carne. As fórmulas utilizadas nos cálculos são as mesmas reportadas no trabalho de Piggott e Marsh (2004) e, portanto, são omitidas. 
Tabela 3. Elasticidades-preço, renda e dos índices agregados de segurança do alimento para o sistema escolhido

\begin{tabular}{ccccc}
\hline \multicolumn{2}{c}{ Elasticidades-Preço Marshallianas } & & \multicolumn{2}{c}{ Elasticidades-Preço Hicksianas } \\
\cline { 1 - 1 }$\eta_{\mathrm{b}, \mathrm{b}}$ & $-0,790$ & & $\varepsilon_{\mathrm{b}, \mathrm{b}}$ & $-0,267$ \\
$\eta_{\mathrm{b}, \mathrm{p}}$ & 0,485 & $\varepsilon_{\mathrm{b}, \mathrm{p}}$ & 0,777 \\
$\eta_{\mathrm{b}, \mathrm{c}}$ & 0,413 & $\varepsilon_{\mathrm{b}, \mathrm{c}}$ & 0,607 \\
$\eta_{\mathrm{p}, \mathrm{b}}$ & 0,627 & $\varepsilon_{\mathrm{p}, \mathrm{b}}$ & 1,236 \\
$\eta_{\mathrm{p}, \mathrm{p}}$ & $-0,657$ & & $\varepsilon_{\mathrm{p}, \mathrm{p}}$ & $-0,304$ \\
$\eta_{\mathrm{p}, \mathrm{c}}$ & 0,161 & $\varepsilon_{\mathrm{p}, \mathrm{c}}$ & 0,409 \\
$\eta_{\mathrm{c}, \mathrm{b}}$ & 0,546 & $\varepsilon_{\mathrm{c}, \mathrm{b}}$ & 0,795 \\
$\eta_{\mathrm{c}, \mathrm{p}}$ & 0,216 & $\varepsilon_{\mathrm{c}, \mathrm{p}}$ & 0,374 \\
$\eta_{\mathrm{c}, \mathrm{c}}$ & $-0,304$ & $\varepsilon_{\mathrm{c}, \mathrm{c}}$ & $-0,180$ \\
\hline
\end{tabular}

Elasticidade Quanto a Segurança do Alimento

\begin{tabular}{|c|c|c|c|}
\hline \multicolumn{2}{|c|}{ Efeito Direto } & \multicolumn{2}{|c|}{ Efeito Total } \\
\hline$\omega_{\mathrm{b}, \mathrm{bf}}$ & $-0,0145$ & $\Psi_{\mathrm{b}, \mathrm{bf}}$ & $-0,0003$ \\
\hline$\omega_{\mathrm{b}, \mathrm{pk}}$ & 0,0047 & $\Psi_{\mathrm{b}, \mathrm{pk}}$ & 0,0074 \\
\hline$\omega_{\mathrm{b}, \mathrm{py}}$ & 0,0090 & $\Psi_{\mathrm{b}, \mathrm{py}}$ & $-0,0119$ \\
\hline$\omega_{\mathrm{p}, \mathrm{bf}}$ & $-0,0582$ & $\Psi_{\mathrm{p}, \mathrm{bf}}$ & $-0,0552$ \\
\hline$\omega_{\mathrm{p}, \mathrm{pk}}$ & 0,0055 & $\Psi_{\mathrm{p}, \mathrm{pk}}$ & $-0,0041$ \\
\hline$\omega_{\mathrm{p}, \mathrm{py}}$ & 0,0460 & $\Psi_{\mathrm{p}, \mathrm{py}}$ & $-0,0035$ \\
\hline$\omega_{c, b f}$ & $-0,0293$ & $\Psi_{\mathrm{c}, \mathrm{bf}}$ & $-0,0277$ \\
\hline$\omega_{\mathrm{c}, \mathrm{pk}}$ & 0,0221 & $\Psi_{\mathrm{c}, \mathrm{pk}}$ & 0,0090 \\
\hline$\omega_{c, p y}$ & $-0,0111$ & $\Psi_{c, p y}$ & $-0,0140$ \\
\hline \multicolumn{4}{|c|}{ Elasticidades-Renda } \\
\hline & $\eta_{b, x}$ & \multicolumn{2}{|c|}{1,008} \\
\hline & $\eta_{p, x}$ & \multicolumn{2}{|c|}{1,210} \\
\hline & $\eta_{c, x}$ & \multicolumn{2}{|c|}{0,532} \\
\hline
\end{tabular}

Nota: $\eta_{\mathrm{i}, \mathrm{j}}$ e $\varepsilon_{\mathrm{i}, \mathrm{j}}$ representam as elasticidades-preço das demandas Marshallianas e Hicksianas para o bem $i$ com respeito a variações no preço do bem $j$, e $\eta_{\mathrm{i}, \mathrm{x}}$ é a elasticidade-renda para o bem $i$, com $i, j=\mathrm{b}$ para carne bovina, p para carne suína, e c para carne de aves. $\omega_{\mathrm{i}, \mathrm{k}}$ mede a mudança percentual na quantidade pré-comprometida com um bem i em resposta a $1 \%$ de aumento no número de notícias sobre segurança do alimento veiculadas para o alimento $k$, em que $k=b f$ para carne bovina, pk para carne suína, e py para carne de aves. $\Psi_{\mathrm{i}, \mathrm{k}}$ mede a mudança percentual na quantidade total consumida para um alimento $i$ em resposta a $1 \%$ de aumento no número de notícias sobre segurança do alimento veiculadas para o alimento $k$. Estimativas apresentadas correspondem a média das elasticidades computadas para cada observação da amostra de dados, utilizando os valores estimados das parcelas do gasto total com carnes efetuado com cada tipo de carne. 
Na Tabela 3, observa-se que as elasticidades-preço Marshallianas para os próprios preços são $-0,790$ para a carne bovina, -0,657 para a suína e -0,304 para a de aves. Tais estimativas indicam que as demandas Marshallianas dos três tipos de carnes são inelásticas a variações nos próprios preços, sendo todos bens comuns (elasticidades-preço Marshalliana para o próprio preço negativa).

As elasticidades-preço cruzado Marshallianas mostram que a demanda por carne suína (veja que 0,627 > 0,485) e de aves $(0,546>0,413$ ) são mais sensíveis a variações nos preços da carne bovina do que o contrário. Além disso, verifica-se que a demanda por carne de aves é mais sensível a variações nos preços da carne suína do que o contrário (veja que 0,216>0,161). De qualquer forma, os três tipos de carne se apresentam como bens substitutos brutos uns dos outros.

Como requerido pela teoria do consumidor, todas as elasticidadespreço Hicksianas para os próprios preços são não-positivas $(-0,267$ para a carne bovina, -0,304 para a carne suína e -0,180 para a carne de aves). Já as elasticidades-preço cruzadas Hicksianas indicam que os três tipos de carnes são substitutos compensados uns dos outros. Tal e qual observado para as elasticidades cruzadas Marshallianas, as elasticidadespreço cruzado Hicksianas indicam que as demandas compensadas por carne suína (veja que $1,236>0,777)$ e de aves $(0,795>0,607)$ são mais sensíveis a variações nos preços da carne bovina do que o contrário. Além disso, verifica-se que a demanda por carne de aves é mais sensível a variações nos preços da carne suína do que o contrário (veja que $0,374>0,607)$.

Avaliando as elasticidades-renda, observa-se que todas as carnes são bens normais (elasticidade-renda positiva), sendo que as carnes bovina e suína são bens de luxo $\left(\eta_{i, x}>1\right.$ para $\left.i=b, p\right)$, ao passo que a carne de aves é uma necessidade $\left(\eta_{c, x}<1\right)$.

Todas as elasticidades totais do efeito de notícias negativas sobre segurança do alimento sobre o consumo da própria carne foram negativas $\left(\Psi_{\mathrm{b}, \mathrm{bf}}=-0,0003, \Psi_{\mathrm{p}, \mathrm{pk}}=-0,0041, \Psi_{\mathrm{c}, \mathrm{py}}=-0,0140\right)$, como esperado. Interessante observar que, das elasticidades totais do efeito de notícias negativas sobre segurança do alimento, apenas duas apresentaramse positivas $\left(\Psi_{\mathrm{b}, \mathrm{pk}}=0,0074\right.$ e $\left.\Psi_{\mathrm{c}, \mathrm{pk}}=0,0090\right)$. Esse resultado indica que notícias sobre a segurança do alimento para a carne suína aumentam 
a demanda final por carne bovina e de aves. Ou seja, o consumidor substitui carne suína por carne bovina e/ou de aves quando se sente inseguro sobre a segurança em se consumir a mesma.

\section{Sobre os benefícios do NAIS}

Se baseando nas estimativas obtidas para o sistema escolhido, nesse ponto, torna-se possível simular o valor econômico do NAIS, considerando-se os seus impactos sobre a confiança do consumidor americano na segurança de cada tipo de carne. Para tanto, é necessário recordar que os modelos foram estimados utilizando três séries de índices agregados de segurança do alimento, um para cada tipo de carne. No processo de criação de cada uma dessas três séries agregadas de índices de segurança do alimento, foram computados primeiramente dois grupos de séries de índices para cada tipo de carne. No primeiro grupo, as séries foram rotuladas de índices de segurança do alimento aparentemente não-relacionados com o NAIS. No segundo grupo, as séries foram chamadas de índices de segurança do alimento relacionados com o NAIS. Finalmente, somando-se essas duas séries para cada tipo de carne s obtém a série agregada de índices de segurança do alimento. Assim, para simular o impacto do NAIS no período entre 1982:4 e 2001:4, procedeu-se a construção de três cenários com base na manipulação dessas séries de índices de segurança do alimento como exposto a seguir.

No cenário-base, assume-se que o NAIS não teria sido implantado em todo o período 1982:4 a 2001:4. Dessa forma, substitui-se as séries de variáveis explicativas nas equações estimadas do sistema escolhido, obtendo-se os valores estimados das parcelas do gasto com carnes despendida com cada tipo de carne. Em seguida, cada uma dessas séries é multiplicada pelo dispêndio per capita com carnes, o que gera as séries de receitas per capita para cada tipo de carne. Essas séries são finalmente multiplicadas pela população total dos EUA e convertidas para dólares de setembro de 2005, utilizando o CPI (consumer price index) para todos os bens, gerando, dessa forma, as séries das receitas com cada carne para o cenário-base.

No Cenário 1, assume-se que o NAIS estaria em uso em todo o período analisado (1982:4 a 2001:4), possibilitando rastrear todo o gado 
bovino dos EUA. Essa hipótese é implementada, substituindo-se nas equações do sistema estimado a série de índices de segurança do alimento não-relacionada com o NAIS para a carne bovina, no lugar da série de índices agregados de segurança do alimento para a carne bovina. Esse procedimento retira o número de notícias contabilizadas na série índice de segurança do alimento relacionado com o NAIS da série agregada, o que exclui o efeito de notícias sobre BSE sobre a demanda por carne bovina. Dessa forma, todo o efeito negativo de BSE relacionado com o consumo de carne bovina estaria sendo eliminado, devido à presença do NAIS nos EUA no período em análise. Assim, inserindo a série de índices de segurança do alimento não-relacionada com o NAIS para a carne bovina e as demais séries das variáveis explicativas dos modelos, obtêm-se as estimativas das parcelas gastas com cada carne em cada período de tempo. Os passos seguintes são idênticos aos adotados no cenário-base para se obter as séries de receita total em valores de setembro de 2005 para cada carne.

No Cenário 2, assume-se que o NAIS teria sido implementado para se rastrear, além de todo o gado bovino, também todo o rebanho suíno dos EUA. Para implementar essa hipótese, substitui-se no lugar das séries agregadas de segurança do alimento para carne bovina e suína, as suas respectivas séries de índices de segurança do alimento nãorelacionadas com o NAIS. Essas duas séries, juntamente com as demais séries de variáveis explicativas, são inseridas nas equações estimadas do sistema escolhido e os passos subsequentes são os mesmos adotados no cenário-base para se obter as séries de receita total em valores de setembro de 2005 para cada carne.

A Tabela 4 apresenta os números e estatísticas essenciais à discussão sobre o valor econômico do NAIS para os setores de carnes bovina, suína e de aves. 
Tabela 4. Mudanças previstas para a receita total para as carnes bovina, suína e de aves entre cenários (1982:4 - 2001:4)

\begin{tabular}{|c|c|c|c|c|c|c|}
\hline & \multicolumn{6}{|c|}{ Diferenças entre as receitas totais em milhões de dólares de setembro/2005 } \\
\hline & \multicolumn{3}{|c|}{ Cenário 1- Cenário-Base } & \multicolumn{3}{|c|}{ Cenário 2- Cenário-Base } \\
\hline & Boi & Porco & Aves & Boi & Porco & Aves \\
\hline Mínimo & $-192,81$ & $-11,18$ & $-229,05$ & $-204,22$ & $-0,47$ & $-211,54$ \\
\hline Máximo & 303,71 & 222,26 & 25,68 & 179,20 & 237,44 & 8,18 \\
\hline Desvio Padrão & 65,92 & 35,70 & 50,72 & 54,11 & 41,48 & 51,83 \\
\hline Moda & 0,00 & 0,00 & 0,00 & 0,00 & 0,00 & 0,00 \\
\hline Total & $1.412,173$ & $1.256,72$ & $-2.240,62$ & $1.048,540$ & $1.882,64$ & $-2.662,16$ \\
\hline Média & 18,34 & 16,32 & $-29,10$ & 13,62 & 24,45 & $-34,57$ \\
\hline
\end{tabular}

Fonte: Cálculos detalhados no texto.

A Tabela 4 apresenta as diferenças entre as receitas totais obtidas nos Cenários 1 e 2 em relação ao cenário-base. Diante disso, observa-se que se o NAIS tivesse sido implementado somente para o gado bovino (vide colunas de 2 a 4 da Tabela 4), os setores produtores de carne bovina e suína teriam experimentado um aumento médio respectivamente de 18,34 e de 16,32 milhões de dólares por trimestre, enquanto o setor produtor de carne de aves teria experimentado uma perda média de US\$ 29,1 milhões por trimestre. Observe que existe um efeito positivo do aumento da confiança dos consumidores em consumirem carne bovina sobre o consumo de carne suína ${ }^{5}$. Isso ocorre porque, possivelmente, o consumidor americano considera a carne bovina mais parecida com a suína do que com a de aves. De fato, sabe-se que as carnes bovina e suína são dois exemplos de carne vermelha, enquanto a carne de aves, como o peru e frango, é classificada como branca. Dessa forma, mesmo que BSE não possa ser transmitida pela carne suína, o consumidor final pode ficar receoso com relação à BSE na carne suína, devido às similaridades dessa carne com a bovina.

Já se o NAIS estivesse implementado em todo o período para bovi-

${ }^{5}$ Vide a Tabela 3 para ver que esse resultado era esperado, uma vez que uma redução percentual no número de notícias sobre a segurança da carne bovina impactaria positivamente a demanda final por carne suína, segundo a elasticidade $\Psi_{\mathrm{p}, \mathrm{bf}}=-0,0552$. 
nos e suínos (Cenário 2), esses setores teriam aumentado a sua receita média por trimestre respectivamente em US\$13,62 e US\$24,45 milhões. No entanto, o setor de aves teria perdido, em média, US $\$ 34,57$ milhões por trimestre.

\section{Conclusão}

Estimativas preliminares dos custos do NAIS para os rebanhos bovino e suíno são de US $\$ 550$ milhões para um período de cinco anos (Gray, 2004), o que representaria um custo de US\$27,5 milhões por trimestre para cada um desses setores. Esse montante é menor do que os US \$38,07 milhões que é a soma dos ganhos em receita por trimestre para a carne bovina e suína no Cenário 2, o que indica que o NAIS para bovinos e suínos poderia ser pago com os ganhos em receita. Contudo, é importante ressaltar que, para tanto, deve se confirmar a premissa adotada no presente trabalho, segundo a qual, todo e qualquer receio do consumidor em relação à BSE associada ao consumo de carne bovina e suína seria completamente eliminada com o NAIS em funcionamento.

Sabe-se, ainda, que aumentos das receitas totais obtidas no varejo da carne bovina e suína não informam sobre como esses aumentos chegariam aos produtores agrícolas que são aqueles que, de fato, arcarão com os custos de implementação do NAIS. Ou seja, pode ser que mesmo com os setores das carnes bovina e suína obtendo ganhos suficientes para cobrir os custos da implantação do NAIS, produtores agrícolas acabem por receber uma parcela dos ganhos insuficiente para cobrir os custos da implantação do NAIS. Assim sendo, estudos sobre como potenciais ganhos no varejo seriam transmitidos à cada segmento da cadeia produtiva de cada setor devem ser o objeto de trabalhos futuros.

\section{Referências bibliográficas}

ALSTON, J.M., CHALFANT, J.A., PIGGOTT, N.E. Incorporating Demand Shifters in the Almost Ideal Demand System. Economics Letters, v.70, n.1, p.73-8, 2001. 
BAILEY, D., SLADE, J. Factors Influencing Support for a National Animal Identification System for Cattle in the United States, Denver, CO ed. Denver, CO: American Agricultural Economics Association Annual Meetings, August 1-4, 2004.

BROWN, P., WILL, R.G., BRADLEY, R., ASHER, D.M., DETWILER, L. Bovine Spongiform Encephalopathy and Variant Creutzfeldt-Jakob Disease: Background, Evolution, and Current Concerns. Emerging Infectious Diseases, v.7, n. 1, p.6-16, 2001.

BERNDT, E.R. The Practice of Econometrics: Classic and Contemporary. Cambridge: Addison-Wesley Publishing Company, Inc., 1996. 702 p.

BERNDT, E.R., SAVIN, N.E. Evaluation and Hypothesis Testing in Singular Equation Systems with Autoregressive Disturbances. Econometrica, v. 32, p.937-57, 1975.

DICKINSON, D.L., BAILEY, D. Meat Traceability: Are U.S. Consumers Willing to Pay for It? Journal of Agricultural and Resource Economics, v.27, n.2, p. 348-64, 2002.

FISHER, D., FLEISSIG, A.R., SERLETIS, A. An Empirical Comparison of Flexible Demand System Functional Forms, Journal of Applied Econometrics, v.16, n.16, p. 59-80, 2001.

GRAY, C.W. The National Animal Identification System: Basics, Blueprint, Timelines, and Processes, In: Doyle, H. (Ed.), U.S. Livestock Identification Systems: Risk Management and Market Opportunities. Western Extension Marketing Committee, Tucson, USA, p. 1.1-1.11, 2004Available at http://www.lmic.info/memberspublic/animalID/fs01.pdf

HOBBS, J.E. Consumer Demand for Traceability. Working Paper No. 03-1, International Agricultural Trade Research Consortium, 2003. Available at http://www.agtradepolicy.org/output/resource/CI2.pdf

ITALIANER, A. A Small-Sample Correction for the Likelihood Ratio Test. Economics Letters, v.19, n.4 , p. 315-17, 1985.

LEXIS-NEXIS. Search engine, 2005. Available at http://web.lexis-nexis. com.floyd.lib.umn.edu/universe/ 
MOSCHINI, G., MORO, D., GREEN, R. Maintaining and Testing Separability in Demand System. American Journal of Agricultural Economics, v.76, n.1, p.61-73, 1994.

NARDONE, A. Impact of BSE on Livestock Production System, Veterinary Research Communications, v. 27, Supplement 1, p. 39-52, 2003.

PIGGOTT, N.E., MARSH, T.L. Does Food Safety Information Impact U.S. Meat Demand? American Journal of Agricultural Economics, v.86, n.1, p.154-74, 2004.

QUANTITATIVE MICRO SOFTWARE. EViews 5 Command and Programming Reference. Irvine, USA: Quantitative Micro Software, 2004. 670 p.

SOUZA-MONTEIRO, D.M., CASWELL, J.A. The Economic of Implementing Traceability in Beef Supply Chains: Trends in Major Producing and Trading Countries, Working Paper No. 2004-6, ed. Amherst, MA: University of Massachusetts Amherst, Department of Resource Economics, 2004.

USDA/ERS, 2005a. Red Meat Yearbook. Available at http://usda. mannlib.cornell.edu/data-sets/livestock/94006/.

USDA/ERS, 2005b. Poultry Yearbook. Available at http://usda.mannlib.cornell.edu/data-sets/livestock/89007/. 